\title{
Pulmonary embolism: A brief review
}

\author{
Harshita Arun Pardhe ${ }^{1 *}$, Hariprasad MG $^{2}$, Nagalakshmi NC ${ }^{3}$, Prabhat Kumar Chourasia ${ }^{4}$, Pallavi ${ }^{5}$ \\ ${ }^{1,3-5} \mathrm{M}$ Pharm, ${ }^{2} \mathrm{PhD}$, Dept. of Pharmacology, ${ }^{1,3-5}$ Mallige College of Pharmacy, Bengaluru, ${ }^{2}$ Karnataka, KLE University College of \\ Pharmacy, Belgaum, Karnataka, India
}

*Corresponding Author: Harshita Arun Pardhe

Email: pardhehari97@gmail.com

\begin{abstract}
Pulmonary embolism (PE) is characterized as pulmonary artery obstruction caused by a thrombotic embolus the usual clinical presence of acute PE involves a patient with a sudden onset of dyspnea and pleuritic chest pain at breathing, while blood coughing (hemoptysis), signs of deep vein thrombosis (DVT) and even collapse are less prevalent. PE is classified as PE which is massive, sub-massive and low risk. PE is a significant cause of morbidity and mortality among the patients who are hospitalized. When venous clot is dislodged from the place where it was actually formed, the clot then proceeds towards a vessel of pulmonary circulation leading to pulmonary embolism. Many factors affect pulmonary embolism such as prolonged immobility, advanced age, postoperative time, post-infarction period, heart failure, obesity, pregnancy, etc. Treatment mainly involves anti- coagulants. This brief review summarizes the pathophysiology, risk factors and treatment, management and supportive measures involved in Pulmonary embolism.
\end{abstract}

Keywords: Pulmonary Embolism, Deep vein thrombosis, Bronchospasm, Thromboembolism.

\section{Introduction}

Pulmonary embolism is an acute, severe condition which can be a direct threat to life. It happens when a lung artery is blocked by a substance that has traveled through the blood stream from elsewhere in the body. This substance is usually the result of a clot of blood in the legs or pelvis. ${ }^{1} \mathrm{PE}$ is a life-threatening and dramatic complication of deep venous thrombosis (DVT). For this reason, DVT's prevention, diagnosis and treatment is of particular importance as symptomatic PE occurs in $30 \%$ of those affected. If asymptomatic symptoms are also included, 5060 percent of DVT patients are expected to experience PE. ${ }^{2}$ DVT and PE as manifestations of the same cause, namely thrombo embolic disease. PE is a significant cause of morbidity and mortality among hospitalized patients. ${ }^{3}$ The worldwide prevalence of acute venous thromboembolism ranges from 23-69/100,000 population per year. Close to 10 percent of all patients with acute PE die during first 3 months after diagnosis. ${ }^{4}$ Though the exact epidemiology of PE in India is largely unknown, an autopsy study showed the overall incidence of PE in patients admitted in the medical wards of a tertiary care centre in North India to be 15.9 percent, mainly affecting younger population below 50 years of age. The rate of significant PE leading to patients ' death was $12.6 \% .^{3}$

\section{Classification of pulmonary embolism:5}

The American Heart Association classifies PE into three categories

Massive PE: Acute PE with prolonged hypotension (systolic blood pressure $<90 \mathrm{~mm} \mathrm{Hg}$ for at least 15 minutes or needing inotropic treatment, not for causes other than PE such as arrhythmia, hypovolemia, sepsis or left ventricular dysfunction), with signs or symptoms of shock.

Sub-massive PE: Acute PE without systemic hypotension (systolic blood pressure $>90 \mathrm{~mm} \mathrm{Hg}$ ) but with either right ventricular (RV) dysfunction or myocardial necrosis dysfunction. RV dysfunction means the presence of at least one of the following:

1. RV dilation (apical 4-chamber RV diameter divided by LV diameter $>0.9$ ) or RV systolic dysfunction on echo cardiography or CT

2. Elevation of BNP $(>90 \mathrm{pg} / \mathrm{ml})$ or

3. Elevation of $\mathrm{N}$ - term in pro-BNP $(>500 \mathrm{pg} / \mathrm{ml})$ or

4. ECG changes (new right bundle-branch block, anteroseptal ST elevation or depression, or T-wave inversion)

5. Elevation of troponin I $(>0.4 \mathrm{ng} / \mathrm{ml})$ or troponin $\mathrm{T}(>0.1$ $\mathrm{ng} / \mathrm{ml}$

Low risk PE: Acute PE and lack of adverse prognostic clinical markers defining massive or submassive PE.

\section{Pathophysiology: ${ }^{1}$}

If the venous clot is dislodged from the place in which it was created, then its embolism proceeds towards a vessel of pulmonary circulation or systemic arterial circulation. (Paradoxical embolism, seen in open foramen ovale cases) Experimentally, it has been shown that if 60 percent of the vascular network of the pulmonary artery is blocked, then a significant drop in blood pressure and an acute bend of the right ventricular (acute or pulmonal) is triggered. Sudden death occurs in situations where the blockage crosses 80 percent of the vascular network. Cardiac hemodynamic effects on pulmonary embolism depend on the occlusion rate of the pulmonary vasculature, the distance of this network and the release of bronchospasm-causing vasomotor substances followed by a further reduction in pulmonary perfusion and an increase in VD (dead place). Acute pulmonary heart is caused by the sudden increase in the average pulmonary artery pressure due to increased pulmonary vascular resistance in concentrations greater than $40 \mathrm{mmHg}$. The symptoms of coronary pulmonary embolism can include acute pulmonary stroke, myocardial ischemia, acute circulatory failure, and left-sided and lung and hypoxia and atelectasis. 
The pressure in the right ventricle rises in an acute deficit, resulting in the displacement of the septum to the left, causing both ventricles to malfunction, reduction in cardiac output, and a drop in blood pressure. Coronary insufficiency due to pulmonary embolism is attributed to significant reduction in cardiac output, decrease in the pressure of the aorta, hypoxia, and likely to the presence of a vagal reflex that decreases the width of the coronary arteries.

Pulmonary infarction appears as a thickening hemorrhagic lung area when pulmonary arteries of average size are unblocked due to left heart failure and drop in systemic circulation pressure. Pulmonary infarction occurs only in 10 percent of cases of pulmonary embolism because bronchial oxygenation of the pulmonary parenchyma is sufficient. The condition of the thromboembolism in the lung will decide the disease's further course. Some mechanisms restore pulmonary artery drainage, unless the events replicate themselves. If an oversized embolous plugs in the pulmonary circulation can cause transient syncope, but it can then be destroyed and dissolved within a few 24 hours, otherwise it will be organized, shrink and coalesced with the vessel's wall and the bronchial arteries. A few days after the insertion of the embolus, the process of resetting the damage by fibrinolytic processes through the lysis of the thrombus starts and eventually the damage is arranged in such a way that the clot is transformed into a small scar adhering to the vascular wall. The cycle is done in 10-14 days. In cases where the restoration of circulation, given the above mechanisms, delays, the maintenance of collateral bronchial movement restores the flow in the capillary network resulting in a gradual restoration of surfactant output, atelectasis, hypocapnia and vasoconstriction.

\section{Risk factors: ${ }^{6}$}

Virchow's suggested thromboembolic disease risk factors in 1856- local vascular wall damage, increased coagulability, and circulatory stasis - can explain most cases of DVT and PE. Long-term immobility, advanced age, heart failure, obesity, post-operative, post-infarction, pregnancy, and other factors predispose for thromboembolic disease by venous stasis. Events such as local trauma, vasculitis, and prior thrombosis damage the venous wall's endothelium. Polycythemia, malignant cancer, contraceptive pills, and adenocarcinomas in particular, are associated with coagulability disorders and increased risk of DVT and PE.

\section{Treatment}

\section{Heparin}

Heparin linked with antithromvoni III increases its own activity. It prevents the current thrombus from increasing in size and encourages the breakdown of the endogenous fibrinolytic network. Between 5-7 days of heparin administration, thrombus stabilisation occurs in vein endothelium or pulmonary artery. Heparin doesn't remove the current clot. ${ }^{7}$

Dosage: The initiation of heparin care is after patient monitoring of the presence of a severe bleeding site and after rectal examination, in order to rule out microscopic bleeding from the digestive tract. Initially, 5000-10000 units of the drug are given in a single dose and 1000-1500 units per hour are subsequently administered by continuous intravenous injection. Heparin therapy levels are achieved when the duration of partial thromboplastin time for the patient is at least twice that of the monitor. ${ }^{8}$

Complications: Bleeding is the primary side effect of heparin. If life threatening intracranial bleeding occurs, the drug is discontinued and protamine sulfate is given. 1 Osteoporosis, osteopenia and pathological bone fractures can occur in patients undergoing long-term treatment with heparin. There are often irregular liver function tests but they are rarely associated with clinically significant toxicity. ${ }^{9}$

\section{Warfarin}

Warfarin A vitamin $\mathrm{K}$ antagonist, it prevents the activation of coagulation factors II, VII, IX and $\mathrm{X}$ by $\mathrm{c}$ decarboxylation. Often a duration of five days is required for the manifestation of its full effect, even if the prothrombin time (through which the action is controlled) is extended in a shorter time. When its administration starts during the active thrombotic state, protein levels $\mathrm{C}$ and $\mathrm{S}$ are reduced, thus promoting thrombogenesis. The concurrent use of heparin for a duration of five days compensates this warfarin action. ${ }^{10}$

Dosage: It is initially given at a dosage of $7.5-10 \mathrm{mg}$. Usually however, the dose should be reduced after a period of some days. In patients with poor nutritional status, or patients who have received long-term antibiotic treatment and may exhibit some degree of avitaminosis $\mathrm{K}$, the initial dose of warfarin should be significantly lower, e.g. 2.5 mg. ${ }^{10,1}$

Complications: The most common complication as with heparin is bleeding. When life-threatening bleeding occurs, freshly frozen plasma is administered (normally two points) to maintain hemostasis. ${ }^{7}$ The risk of recurrence of pulmonary embolism after stopping treatment with anticoagulants is extremely high. Patients with cancer or extreme obesity are likely to take anticoagulants for their entire lives. Anticoagulation is prescribed for the other patients for a single episode of thrombosis of the leg veins for three months, an in the vein thrombosis of the proximal venous leg network for 6 months and pulmonary embolism for 1 year. ${ }^{1,11}$

\section{Thrombolysis}

The American College of Chest Physicists (ACCP) recommends systemic thrombolysis for severely compromised patients if systolic blood pressure is < 90 mmHg. ${ }^{12}$ The American Heart Association also reports that ' fibrinolysis is appropriate for patients with major acute pulmonary embolism and an acceptable risk of bleeding complications This includes patients with systolic blood pressure $<90 \mathrm{mmHg}$ or bradycardia $<40$ beats / minute, and fibrinolysis may be considered for... submassive acute pulmonary embolism (with)... hemodynamic instability, 
worsening breathing insufficiency, severe right ventricular dysfunction, or major myocardial necrosis and low risk of bleeding complications. ${ }^{5}$

\section{Embolectomy $^{13}$}

Transvenous catheter thrombectomy is performed in patients with haemodynamic instability in whom thrombolysis has failed or is contraindicated (intracranial haemorrhage, recent surgery or trauma). Various devices for the aspiration or pulverization of thrombus in the pulmonary circulation have been developed. If such a device is not available or the procedure fails, the removal of surgical thrombus with open tho-racotomy and extracorporeal circulation is indicated.

While emergency embolectomy has found widespread acceptance, the results are unsatisfactory as the condition of patients referred for surgery is extremely severe and reported 58 percent death among patients undergoing emergency embolectomy who have experienced car-diac arrest. Additional risk factors are cardiac arrest and cardiogenic shock. Rapid diagnosis and haemodynamic stabilization play a decisive role in the improvement of outcomes.

\section{Maintenance treatment: ${ }^{14}$}

The risk of recurrence of thrombosis is particularly high in patients who were unprovoked by the initial venous thrombosis. PE's recurrence rate within 5 years is 25 per cent. There are several high-risk factors for recurrent $\mathrm{PE}$, including a history of $\mathrm{PE}$, male gender, proven protein $\mathrm{S}$ and $\mathrm{C}$ deficiencies, factor $\mathrm{V}$ and prothrombin G20210Ahomozygosity, lupus anticoagulanone thrombosis in the proximal veins, and active cancer. Such patients become eligible after the first unprovoked PE attack for treatment of unspecified duration. The risk of hemorrhage should not be forgotten in patients undergoing secondary prophy-laxis.

In patients with triggered $\mathrm{PE}$, three months of anticoagulant therapy is appropriate as long as underlying reversible risk factors such as surgical intervention, trauma, medical illness, estrogen medication and pregnancy are eliminated.

\section{Supporting measures: ${ }^{1}$}

These measures include pain relief (mainly the administration of anti-inflammatory nonsteroidal agents), supplementary oxygen therapy, and psychological assistance. Dobutamine (beta-adrenoceptor agonist with positive inotropic activity and vasodilator activity in pulmonary circulation) is administered for the treatment of right heart failure and cardiogenic shock. In general, intravenous fluid loading should be avoided as the dilatation of the right ventricle is exacerbated and may result in further reduction of the supply of the left ventricle.

\section{Conclusion}

Pulmonary embolism is the most severe chronic acute fatal disease and most of it remains undiagnosed, which has a negative impact on survival, thereby requiring timely diagnosis and treatment due primarily to relapses. Following the initial PEattack, risk factors should be carefully investigated and management should be planned accordingly.

\section{Acknowledgement}

My sincere gratitude goes to Mrs N.C Nagalakshmi, Dr. Hariprasad M.G and friends Mallige college of pharmacy Bangalore for their constant support and guidance

\section{Source of funding}

None.

\section{Conflict of interest}

None.

\section{References}

1. Lambirini K, Konstantinos K, Christos I, Petros O, Areti T. Pulmonary Embolism: A Literature Review. Am J Nurs Sci 2018;7(3-1):57-61.

2. Moser KM, Fedullo PF, LitteJohn JK, Crawford R. Frequent asymptomatic pulmonary embolism in patients with deep venousthrombosis. JAMA 1994;271:223-5.

3. Kakkar N, Vasishta RK. Pulmonary embolism in medical patients: an autopsy-based study. Clin Appl Thromb Hemost 2008;2:159-67.

4. Lankeit M, Konstantinides S. Mortality risk assessment and the role of thrombolysis in pulmonary embolism. Crit Care Clin 2011;4:953-67.

5. Jaff MR. Management of massive and submassive pulmonary embolism, iliofemoral deep vein thrombosis, and chronic thromboembolic pulmonary hypertension: A scientific statement from the American Heart Association. Circ 2011; 123:1788-1830.

6. Anderson FA Jr, Spencer FA: Risk factors for venous thromboembolism. Circ 2003;107:109-16.

7. Mismetti P, Bertoletti L. Initial management of venous thromboembolism. Rev Prat 2015; 65(2):193-8.

8. "Pulmonary Embolism" July 23 2017. Available at www.iatrikionline.gr cited on $24 \mathrm{dec} 2019$

9. Tsilimidos G, Marinis A. Review of the Diagnostic and Therapeutic Approach of Pulmonary Embolism. Epistimonika Xronika 2013;18(2):88-91.

10. Girtovitis F. Prevention and treatment of thromboembolic disease in thrombophilic patients. Haema 2014;5(1):41-53.

11. Banovac F. Reporting Standards for Endovascular Treatment of Pulmonary Embolism. J Vasc Interv Radiol. 2010; 21:4453.

12. Kearon C. et al. Antithrombotic therapy for VTE disease: CHEST guideline and expert panel report. Chest 2016; 149(2): 315-52.

13. Kleni Kostadima, Epaminondas Zakynthinos. Pulmonary Embolism: Pathophysiology, Diagnosis, Treatment. Hellenic J Cardiol 2007;48:94-107.

14. Serap Duru, Arif Kelesoglu, Sadik Ardic. Clinical update on Pulmonary Embolism. Arch Med Sci 2014;10(3): 557-65.

How to cite: Pardhe HA, Hariprasad MG, Nagalakshmi NC, Chourasia PK, Pallavi N, Pulmonary embolism: A brief review. IP Int J Comprehensive Adv Pharmacol 2020;5(1):68. 